D:INsurg|Vol. 24, No. 1, Jan. - Mar., 2020\Nsurg-12.Doc $\quad$ P. $15-20 \quad$ III

ORIGINAL ARTICLE

\title{
Navigating Brain Lesions Via Craniometric Points of Human Skull. Effectiveness in Targeted Craniotomy
}

\author{
SHAMS-UD-DIN, ISHFAQ AHMED \\ Department of Neurosurgery, Quaid-e-Azam International Hospital, Islamabad-Pakistan
}

DOI: 10.36552/pjns.v24i1.417

\begin{abstract}
Background and Objectives: Navigating brain pathologies via external landmarks helps in guiding neurosurgeon to perform and plan brain surgeries without feeling compromised in their competence, when modern neuronavigation is not available. We assessed the navigating art of human craniometry in brain surgery, in terms of accuracy and safety, of our patients.
\end{abstract}

Materials and Methods: A descriptive study was in Department of Neurosurgery Quaid-e-Azam International Hospital on utilizing the art of Craniometry in navigating brain lesions and to see how effective this art is in the execution of neurosurgical procedures.

Results: Total of hundred patients were subjected in our study, who were according to inclusion criteria. Mean age calculated was 44 years with STD \pm 6 , with minimum age of 8months and maximum age of 83years. There were $61 \%$ male, and 39\% female patients. Supratentorial lesions were $72 \%$, ventriculoperitoneal shunts $11 \%$, and $17 \%$ were infratentorial.In 89 surgeries, we were exactly on the target according to our external skull landmarks, in 4 cases we slightly deviated about $0.3 \mathrm{~cm}$, and in 7 surgeries we encounter some difficulty in navigating lesions.

Conclusion: Modern navigating technology is very much helpful in neurosurgery, and this fact is undeniable, but because of resources restrained is not always available. Mastering the art of craniometery of human skull guide neurosurgeons to perform/plan neurosurgical procedure of brain without feeling compromised in their competence.

Keywords: Craniotomy, Craniometric points, Human Skull, Craniometery.

Abbreviations: Craniometric points, Frontotemporal key point (FTP), Anterior silvian point (ASP), Superior Rolandic point (SRP), Inferior Rolandic point (IRP), Superior frontal sulcus (SFS), Inferior frontal sulcus (IFS), Pre Central Sulcus (PCS) Computed Tomography (CT) and Magnetic Resonance Imaging (MRI).

\section{INTRODUCTION}

The integration of neuronavigation in modern neurosurgery has revolutionized the surgical practice on human brain. We assessed the navigating art of human craniometry in brain surgery, in terms of accuracy and safety, of our patients. Neuronavigation is extremely helpful in the safe, precise, accurate and targeted execution of surgical lesion of human brain. ${ }^{1,3,4}$ But, this advance technology is not always available, because of resources restrained..$^{2,4}$ Therefore, it is extremely mandatory for neurosurgeons to know the precise anatomical knowledge of cranial external surgical landmarks to approach brain lesions, with safety and accuracy in targeting brain lesions ${ }^{1,4}$. The main objective of this was to target brain lesions according to craniometric points. We reviewed our pre-operative surgical landmarks and per-operative targeted lesions.

The craniometric points of human skull are extremely helpful for establishing topographical anatomy of brain pathology, and guide neurosurgeons for anatomical placement of craniotomy precisely and

-15- Pak. J. of Neurol. Surg. - Vol. 24, No. 1, Jan. - Mar., 2020 
safe. $^{1,2}$ These points in addition to the main sutures will define a framework on skull. ${ }^{2,3,5}$ To facilitate intraoperative identification of pathology, it is mandatory to establish cortical key points for important sulci, gyri anatomical localization via external craniometric points. Interpretation of brain topographical anatomy, radiology, including CT brain, and MRI brain is mandatory for safe, accurate and precise execution of brain surgery. $2,5,7$ Navigating brain pathologies via external landmarks helps in guiding neurosurgeon to perform and plan brain surgeries without feeling compromised in their competence, when modern neuronavigation is not available.

From the frontal view in the human skull, the three craniometric points are, nasion, bregma, and frontozygomatic sutures. The distance from nasion to the bregma is near $13 \mathrm{~cm} .{ }^{1,5,7}$ From the upper view, the coronal suture, the sagittal sutures and the lambdoid sutures. ${ }^{2,5,7}$ The lambda is located at a distance of $25 \mathrm{~cm}$ posterior to nasion and $13 \mathrm{~cm}$ posterior to bregma. The bregma is coronal suture intersecting with sagittal suture..$^{3,8,9}$ The staphenion is coronal suture intersection with Superior Temporal line. The junction of frontosphenoid suture, squamosal, parietal and coronal suture is called pterion, which lies at the greater sphenoid wing lateral end ${ }^{3,5}$. The parstriangularis portion of the inferior frontal gyrus lower end is situated under this craniometric point. At the lateral orbital rim, above the zygomatic arch at a distance of $2.5 \mathrm{~cm}$ is the craniometric point of frontozygomatic suture. The zygomatic suture is situated at the lateral orbital vim. The Frontozygomatic rim is situated $2.5 \mathrm{~cm}$ above the level of zygomatic arch. ${ }^{5,7}$

The squamosal suture separates the temporal bone. The junction of the temporal lobe middle and posterior $3^{\text {rd }}$ is followed by the posterior limb of the silvian fissure at its anterior portion. ${ }^{1,3,9}$

In the posterior part of the skull, we must localize three craniometric points, in addition to the lambdoid suture and the lambda, we must know the inion, the opisthocranium, the asterion, the superior nuchal line. ${ }^{3,6,7,9}$

The lambda is the point where the sagittal suture and the lambdoid suture join. The Opisthocranium corresponds to the most prominent occipital landmark, and in the superior nuchal line, there is a bony prominence which is the site for inion. ${ }^{1,5,8}$ The lambda was found to be 2 or $4 \mathrm{~cm}$ superior to the opisthocranium, and the inion is located $6-8 \mathrm{~cm}$ inferior to lamba. ${ }^{1,4,6,7}$ The transverse sinus underlies in most cases with these lines. Torcula aerophilae will be located superior to inion. ${ }^{5-8}$ In majority of the cases torcula aerophilae is situated between $5-10 \mathrm{~mm}$ superior to the inion, except in patients with dandy walker malformation or chiari malformation, these landmarks are not optimal. ${ }^{5,6}$ The lambdoid suture provide a rough orientation of the junction of occipital and posteriorly with temporal lobe ${ }^{1,3}$. The asterion is defined as the junction of the lambdoid, parietal, mastoid, and occipitomastoid sutures and is also known as the Marcellus point, because it is similar to the Marcellus slogan. ${ }^{89}$ This craniometric point is an important landmark for approaches to the posterolateral cranial base.Positioning the burrhole away from the asterion reduce the risk of bleeding. Burrhole position is $2 \mathrm{~cm}$ below the asterion and $1 \mathrm{~cm}$ behind and $1 \mathrm{~cm}$ in front of the occipitomastoid suture. ${ }^{8,9}$

The three craniometric points, i.e, frontozygomatic point, nasion, and inion, can define the site of silvian fissure. ${ }^{1,5,8,9}$ First, draw a line through sagital suture from inion to nasion, and divide this line into 2 quarter and 3 quarter. A line extended from frontozygomatic point in the backward direction define the location of silvian fissure on the lateral surface of skull to the 3 quarter point of inion nasion line on sagittal suture. ${ }^{5,8,9}$

Location of superior rolandic point can be noted behind the bregma at approximately $5 \mathrm{~cm}$. Approximating central sulcus is done by connecting line from superior rolandic point and inferior rolandic point. On the silvian fissure line, at approximately $2.5 \mathrm{~cm}$ posterior to pterion is the location of lower rolandic point. ${ }^{5,7-9}$ At approximately $5 \mathrm{~cm}$ posterior to bregma, is the precentral gyrus. Identifying postcentral gyrus topography is posterior to bregma at $6.5 \mathrm{~cm}$ and $4 \mathrm{~cm}$ posterior to staphenion. ${ }^{6-9}$ During craniotomy for specific reason, posterior ramus is not identified, than external ear canal topographically guide to supramarginal and angular gyrus, as these are located just posterosuperior to external ear canal..$^{7-9}$ The anterior silvianpointis called anterior squamosal point, and is located behind pterion on the squamous suture anterior portion. ${ }^{2,3,7,9}$ The Hassel gyrus is at the inferior rolandic point, and resection of inferior and superior temporal in the dominant hemisphere can cause dysphasia, which is permanent, because it corresponds to Wernicke's area. ${ }^{4-6}$ In relation with cranium, the inferior rolandic point is situated at the area, where vertical line drawn from just infront of tragus meet squamous suture. ${ }^{4-6}$ The intersection of 
coronal suture with the superior temporal line is the craniometric point, called staphenion. ${ }^{2,3,4,7}$ The precentral sulcus and inferior frontal sulcus junction is at $1-2 \mathrm{~cm}$ posterior to this area. Inferior to lambda at $2-4 \mathrm{~cm}$ is the calcarine sulcus, approximately where parieto occipital sulcus arise to the occipital external fissure. ${ }^{3,4,7}$

\section{MATERIAL AND METHODS}

\section{Study Design}

This descriptive study was done in the department of Neurosurgery Quaid-e-Azam International hospital, Islamabad, from April 2016 to July 2018. Patients were selected according to the inclusion criteria. Craniotomy was planned according to specific pathological lesion, and navigated via external craniometric landmarks.

\section{Inclusion Criteria}

Patients who were admitted for craniotomy with brain lesions, including brain tumors, spontaneous intracerebral bleed who were surgical candidates for haematoma evacuation, and patients for V-P shunt for any cause of hydrocephalus.

\section{Excluding Criteria}

Patients with traumatic brain injury who undrwent craniotomy for extra dural haematoma, acute subdural haematoma, chronic subdural haematoma and also patients who required decompressive crianiotomy for any reason of raised intracranial pressure.

\section{Data Collection}

Strict inclusion criteria was followed for the selection of patients in this study. All patients and their family were fully informed about the pattern of study, and in writing informed consent were taken. The brain lesions were diagnosed on the basis of history, examination, and radiology in the form of CT brain, and MRI brain. After diagnosis of brain lesions, CT brain and MRI brain were extensively studied anatomically and possible type of lesions, and then were subjected them to the art of human skull craniometry.

\section{Surgical Procedures}

All those patients who were included in the study were subjected to the craniometric points, and were operated under general anesthesia. After GA, patient's positions were made according to the site of lesions, and according to planned craniotomy. Craniometric points were labeled and lesions were navigated according to the external landmarks on skull, and labeled for desired craniotomy. Patients were than cleaned with pyodene scrub solution and then washed with spirit to decrease surgical site infection. Draping also done according to standard protocol. Before skin incision, we infiltrate diluted local anesthesia with adrenaline to help in haemoestasis and also subgaleal dissection. After periosteom is being elevated, key burr-hole for desired craniotomy made, and then craniotomy was done with drill. After dural inspection macroscopically for any signs of pathology, dura opened with duratome and dural cutting scissors according to standard protocol. Per-operative our trajectory on navigating the external landmarks on craniometric points were assessed and recorded. As we identified the lesions intracranial, was proceeded with resections according to standard protocols. It was our protocol to close dura watertight primarily, or if not possible, than grafts were substituted. Bone replaced and wound to closed in layers on standard set protocols.

As our main objective was to target brain lesions according to craniometric points. We reviewed our pre-operative surgical landmarks and per-operative targeted lesions. No per operative CT/MRI guidance or any neuronavigation were used.

\section{Data Analysis}

We assess our craniometric navigation technique in target localization intracranially, and labeled as accurate, when trajectory of our technique was exactly on pathological lesion, and not accurate when we missed target on craniometric landmarks.

\section{RESULTS}

\section{Age \& Gender Distributions}

The mean age calculated was 44 years with STD \pm 6 . Minimum age of patient in our study was 8 months and maximum age 83 years (Table 1). There were 61 male $(61 \%)$, and 39 female $(39 \%)$ patients.

\section{The Outcome}

Supratentorial lesions were $83 \%$, and $17 \%$ were infratentorial. Types of pathologies were, Meningioma $12 \%$, supratentorial intra-axial lesions $31 \%$, metastatic 
brain tumors 2, pituitary tumors $2 \%$, craniopharyngioma $2 \%$, spontaneous intracerebral haematomas, which were surgical candidates according to standard protocols were $18 \%$, posterior fossa including cerebellopontine angle 17\%. Total ventriculoperitonial shunts were $11 \%$, of which 3 were for post infective hydrocephalus, 1 post traumatic hydrocephalus, and 7 were of congenital hydrocephalus. Third ventricular colloid cysts were 4 and $4^{\text {th }}$ ventricular ependymoma 1 (Table 1 ).

Table 1: Brain lesions according to site of pathology.

\begin{tabular}{|l|c|}
\hline Location of Lesions & Percentage \\
\hline Supratentorial intra-axial & 31 \\
\hline Meningioma & 12 \\
\hline Sellar suprasellar lesions & 2 \\
\hline Craniopharyngioma & 2 \\
\hline Brain Mets & 2 \\
\hline $\begin{array}{l}\text { Spontaneous intracerebral bleed who were } \\
\text { surgical candidates according to set protocols. }\end{array}$ & 18 \\
\hline Ventriculoperitonial shunts & 11 \\
\hline $3^{\text {rd }}$ ventricular colloid cyst & 4 \\
\hline $4^{\text {th }}$ ventricular ependymoma & 17 \\
\hline Posterior fossa lesions & 17 \\
\hline
\end{tabular}

In 89 surgeries, we were exactly on the target according to our topographical skull anatomical landmarks, in 4 cases we slightly deviated about 0.3 $\mathrm{cm}$, and in 7 surgeries we encounter some difficulty in navigating lesion.

\section{DISCUSSION}

The term Craniometry is coined for measurements of skull and external landmarks on skull to accurately navigate brain..$^{1-3}$ The art of navigating brain via skull craniometric points came in late $19^{\text {th }}$ and early $20^{\text {th }}$ century when two new fields of that era, anthropology and phrenology emerged. ${ }^{1,3,7}$ Through basic mathematical concepts and mechanical tools, brain function localization were described. This art of human skull craniometry is now well established and also the modern neuronavigation into specific region of brain was made possible by utilizing this art of craniometery. ${ }^{2,7,9}$ Learning the art of human skull craniometery is considered essential to master upon, as modern neuronavigation is not always available because of many reasons. It is important to establish cortical key points to provide sulci, gyri anatomical topography for the placement of safe, accurate and targeted craniotomy. ${ }^{6-8}$ Expanding this art of craniometery gives roadmap for instrumentation in brain mapping, and cranial localization with great accuracy. ${ }^{4}$

These early methods would describe human skull and for brain function localization via external landmarks. ${ }^{3,4,7}$ Among the pioneers, Paul Broca (18241880), French anatomist, give much contribution to this art of cerebral localization. Broca's work was focused on fixed coordinates along cranium to the cerebral topography. ${ }^{1,3,7}$ It was in 1861, when Broca discover motor aphasia in one of his patient who had lesion in the left inferior gyrus of frontal lobe. ${ }^{1,3,6}$ Through specialized measurement, the anterior of skull and inion and Glabella were specifically identified. The condylio-alveolar plane, developed by Broca, is still widely being used in modern neuronavigation system of brain. ${ }^{1,4,7}$ The surgical craniometery was refined on coordinates that were fixed on cranium which used to help in the topography of the underlying cerebral cortex. ${ }^{7,9}$

It was the emerging of X-rays radiography in the early $20^{\text {th }}$ century, which helped in the identification of central sulcus via Taylor-Haughton technique ${ }^{2,5,8}$. This technique was also than used to puncture lateral ventricles via kocker's, keen's and Frasier's entrypoints. ${ }^{8,9}$ It was the work of Sir David Ferrier (1843 - 1928), who pointed to the very organized motor and sensory cortex of brain. The Kocher's (1841-1917), craniometric devices were very accurate in localizing intracranial topography via external cranial landmarks. ${ }^{4,7,8}$

Tubbs RS, et al, in 2001, conducted a study on the external skull landmark of superior sagittal sinus. ${ }^{7-9}$ It was concluded that, it is situated below the sagittal suture, but slightly deviated to the right about $11 \mathrm{~mm}^{8,9}$. Landeiro, et.al, studiedasterion as surgical landmark for approaching tumors of cerebellopontine angle and, inion as a surgical landmark to identify torculae aerophelea. ${ }^{9}$ These surgical landmarks were found excellent for retrosigmoid and posterior fossa craniotomies. Dandy, in 1918, describe a technique to enter into the lateral ventricle of brain, by using external craniometric points. ${ }^{7,9}$ A line drown from midpupillary line to the coronal suture, $10 \mathrm{~cm}$ posterior to nasion, and $3 \mathrm{~cm}$ away from midline and $1 \mathrm{~cm}$ 
anterior to coronal suture. ${ }^{9}$ This technique is now standard to approach lateral ventricles and foramen of monro and even third ventricle. 1,3,7, $^{1,7}$ The catheter is advanced by using the external landmarks, i.e. external auditory meatus and the medial side of canthus to reach the lateral ventricle, which is approximately $4 \mathrm{~cm}$ from cerebral cortex. ${ }^{1,5}$ Through this technique it was made possible that modern endoscope can be used for $3^{\text {rd }}$ ventricular pathologies and also endoscopic $3^{\text {rd }}$ ventriculostomy for obstructive hydrocephalus. ${ }^{4,5,7,9}$

Recently, Ammar et.al, from Egypt conducted a study to evaluate neuronavigation on surgery for glioma. They included 33 patients in their study, and in 33 patients $(94.2 \%)$ the craniotomy flap was exactly on lesion and in 2 cases $(5.7 \%)$, the trajectory was fault. ${ }^{13}$

Du et al, conducted a study on neuronavigation for brain glioma. 75 patients were operated under neuronavigation, and in 62 patients $(82.7 \%)$ they accurately removed tumor and in 13 patients (17.3) tumor was sub totally resected. ${ }^{10}$

Kurimoto et al, conducted a study on comparing neuronavigation and no neuronavigation on glioma surgery, and found that neuronavigation group tumor resection was significantly higher i.e, $64.3 \%$ compared to no neuronavigation in which $38.2 \%$ tumor resection was successful. ${ }^{11}$

Though neuronavigation is very useful adjunct in brain surgeries, but it is time consuming and need calculation and registration with restricted space of the field of operation. Also during neuronavigation there are concerns on shifting brain during surgery due to tissue deformation and errors in operating calculations. ${ }^{10-12}$

\section{CONCLUSION}

It is undeniable that modern neuronavigation for dealing brain pathologies is extremely accurate and very precise in target localization. But knowledge of human craniometery is equally important for neurosurgeons. As modern technologies may not be available always, these craniometric points of human skull and correct interpretation with CT brain and MRI brain are equally important for successful, accurate and precise surgery on intracranial lessons. They are an excellent work for the hemispheric and interhemispheric localization and for the placement of craniotomies according to the location of intracranial pathological lesions. Craniometric landmarks are extremely useful for approaching periventricular and ventricular lesions and for orientation of the anatomical removal of infiltrative tumor.Also mastering this art of craniometery of human skull guide neurosurgeon to perform/plan neurosurgical procedure of brain without feeling compromised in their competence.

\section{REFERENCES}

1. Demitre Serietis, MD, PhD, FRCSC, and T. Gienn pait, MD. Early craniometric tools as a predecessor to neurosurgical stereotaxis. J Neurosur. 2016; 124: 18671874.

2. Kido DK, LeMay M, Levinson AW, Benson WE. Computed tomographic localization of precentral Gyrus. Radiology, 1980; 135: 373-377.

3. Martin N, Gafton S, Vineula F, Dron J, et al.

4. Gross CG: The discovery of Motor Cortex and its background. J Hist Neurosci. 2007; 16: 320-331.

5. Nicolas S, Guida A, Levine Z. Broca and Charcot's research on Jacques Inaudi: The psychological and anthropological study of a mental calculator. J Hist Neurosci. 2014; 23: 140-151.

6. Scultke E: Theodor Kocher's craniometer, Neurosurgery, 2009; 64: 1001-1005.

7. Sun A, Hou L, Cheshier S, Sedrak M. TSV: The accuracy of topographical methods in determining central sulcus: a statistical correlation between modern imaging data and these historical predications. Cureus 6 i.e. $2014 ; 186$.

8. Day JD, Tschabitscher M. Anatomic variation of the Asterion. Neurosurgery, 1998; 42: 198-199.

9. Tubbs RS, Salter G, Elton S, Grabb PA, Oakes WJ. Sagital suture as an external landmark for the superior sagittal sinus. J Neurosurg. 2001; 94: 985-987.

10. Du, et al. ALA-Glioma Study Group Extent of Resection and Survival in Glioblastoma Multiforme: Identification of an Adjustment for Bias. Neurosurgery, 2003; 62: 564-576.

11. Kurimoto, M., Hayashi, N., Kamiyama, H., Nagai, S., et al. Impact of Neuronavigation and Image Guided Extensive Resection for Adult Patients with Supratentorial Malignant Astrocytomas: A Single Institution Retrospective Study. Minimally Invasive Neurosurgery, 2004; 47: 278-283.

12. Barnett, G.H. Youmans Neurological Surgery 6th Edition Navigation for Brain Tumors. Elsevier, Philadelphia, Ch. 2011; Volume 2 (118): 1293-1299.

13. Mahmoud M. Ammar, Mohamed Mohmoud, Ahmed El Saied, Abou Kreasha, Abd Elhay Mousa. Evaluation of Neuronavigation in glioma surgery. Open Journal of Modern Neurosurgery, 2020; 10 (1): Article ID: 96683, 15 page. 


\section{Additional Information}

Disclosures: Authors report no conflict of interest.

Ethical Review Board Approval: The study was conformed to the ethicalreview board requirements.

Human Subjects: Consent was obtained by all patients/participants in this study.

Conflicts of Interest: In compliance with the ICMJE uniform disclosure form, all authors declare the following.

Financial Relationships: All authors have declared that they have no financial relationships at present or within the previous three years with any organizations that might have an interest in the submitted work.

Other Relationships: All authors have declared that there are no other relationships or activities that could appear to have influenced the submitted work.

Address for Correspondence:

Dr. Shams-ud-Din

Department of Neurosurgery

Quaid-e-Azam International Hospital

Islamabad - Pakistan

Email: asz9-0@hotmail.com

\begin{tabular}{|l|l|l|l|}
\hline \multicolumn{4}{|c|}{ AUTHORSHIP AND CONTRIBUTION DECLARATION } \\
\hline Sr.\# & Author's Full Name & Intellectual/Contribution to Paper in Terms of: & Signature by the \\
author(s)
\end{tabular}

Date of Submission: 15-02-2020

Date of Revision: 14-03-2020

Date of Online Publishing: 31-03-2020

Date of Print: 31-03-2020 